\title{
Carpal Tunnel Syndrome in Elderly Patients
}

\author{
Young San Ko${ }^{1}$, Hee-Jin Yang ${ }^{2}$, Sung Bae Park ${ }^{2}$, Young-Je Son ${ }^{2}$, Kang Min Kim², Sang Hyung Lee ${ }^{2}$, \\ Young Seob Chung ${ }^{2}$ \\ ${ }^{1}$ Department of Neurosurgery, Seoul National University College of Medicine, Seoul, \\ ${ }^{2}$ Department of Neurosurgery, Seoul National University Boramae Hospital, Seoul, Republic of Korea
}

Corresponding author:

\section{Hee-Jin Yang}

Department of Neurosurgery,

Seoul National University Boramae

Hospital, 20, Boramae-ro 5-gil,

Dognjak-gu, Seoul 07061, Republic

of Korea

Tel: $+82-2-870-2303$

Fax: $+82-2-870-3863$

E-mail: nsyangdr@gmail.com

Received: July 12, 2018

Revised: July 30, 2018

Accepted: August 4, 2018

\begin{abstract}
Objective: Carpal tunnel syndrome (CTS) is a common entrapment neuropathy. Differences in the clinical features and treatment outcomes of CTS between elderly and young patients remain debatable. We analyzed CTS in elderly patients to assess the differences in the characteristics of CTS between elderly and younger patients. Methods: We retrospectively analyzed patients who were operated for CTS between January 2010 and December 2016. Patients were diagnosed based on clinical features and neurological findings and operated owing to persistent symptoms refractory to conservative management. Nerve conduction study (NCS) and ultrasonography were performed preoperatively. Endoscopic surgery was performed under local anesthesia. Carpal tunnel pressure (CTP) was measured intraoperatively using the Spiegelberg intracranial pressure monitoring device connected to a parenchymal type catheter. Results: We performed 304 operations - 48 wrists operated were in patients aged $\geq 70$ years. No significant difference was observed in the sex distribution. The Elderly group showed more severe clinical symptoms $(p=0.04)$, a higher frequency of thenar atrophy $(p=0.02)$, NCS grades indicative of more severe disease $(p=0.001)$, and more prominent median nerve swelling $(p=0.04)$. Both groups showed similar CTPs before and after division of the transverse carpal ligament. Elderly patients showed poorer outcomes than those observed in younger patients $(p=0.005)$. Conclusion: Our series demonstrated that elderly patients showed more severe clinical features and findings using supplementary tests. Considering the lack of intergroup differences in CTP, we conclude that the reaction to compression of the median nerve in elderly patients causes more severe clinical, radiological, and electrodiagnostic features.
\end{abstract}

Key Words: Aged; Carpal tunnel syndrome; Pressure; Treatment outcome

\section{INTRODUCTION}

Carpal tunnel syndrome (CTS) is a common entrapment neuropathy. It commonly affects middle-aged women ${ }^{22)}$. Considering the rising aging population, elderly patients with CTS are increasingly being observed in clinical practice. Differences in the clinical presentations, findings of supplementary tests, and treatment outcomes of CTS between elderly and young patients remain debatable $e^{3,7,24,28)}$. We analyzed and compared intergroup differences in clinical characteristics and surgical outcomes of CTS between elderly and young patients. Additionally, we measured the carpal tunnel pressure (CTP) intraoperatively to evaluate the basic pathology of CTS.

\section{MATERIALS AND METHODS}

\section{Patients}

We retrospectively reviewed the electronic medical records of patients who were operated for CTS at our hospital between January 2010 and December 2016. CTS was diagnosed based on clinical features in addition to a nerve conduction study (NCS), electromyography (EMG), and ultrasonography (USG).

Severe symptoms refractory to conservative treatment and/or the presence of thenar atrophy were considered indications for surgery. Patients with secondary CTS such as CTS following trauma or CTS secondary to a mass lesion were excluded from this study. Patients aged $\geq 70$ years were assigned to the elderly group (Group I) and those aged $<70$ years to the young patients group (Group II).

All patients underwent endoscopic surgery performed by a single surgeon (HJY) using Endocarp ${ }^{\text {TM }}$ (Linvatec, Largo, FL, USA) as described in a previous report. In a few patients, the CTP was measured intraoperatively ${ }^{1)}$. Patients who underwent pressure measurements had been previously informed regarding the nature of the study and written informed consent was obtained for measurement. This protocol of pressure measurement was approved by the Institutional Review Board of 
our hospital.

Clinical symptoms were graded between Stage I and V using the historical-objective (Hi-Ob) scale proposed by Giannini et al. () as - I, nocturnal paresthesia only; II, nocturnal and diurnal paresthesia; III, presence of sensory deficit; IV, presence of thenar atrophy with, motor deficit of the median nerve; and $\mathrm{V}$, plegia of the muscles of the median thenar eminence.

\section{Electrophysiological Study and USG}

Preoperative NCS were performed using standard surface stimulation and recording techniques (Nicolet Biomedical Instrument Co., Madison, WI, USA). Skin temperatures were maintained above $31.0^{\circ} \mathrm{C}$. The median sensory nerve conduction velocity was orthodromically measured in the index finger-wrist segment and the median distal motor latency from the wrist $5 \mathrm{~cm}$ proximal to the recording electrode at the abductor pollicis brevis. The severity of CTS was assessed based on the results of NCS using a previously reported neurophysiological classification as: negative, minimal, mild, moderate, severe, and ex- treme ${ }^{17 \text { ). }}$

Preoperative USG of the carpal tunnel was performed using HDI 3000 (Philips Medical Systems, Best, Niederlande), and Sequoia 512 (Acuson, Mountain View, CA, USA) by a radiologist who was blinded to the clinical findings. A 2-6 MHz linear array transducer was used. The cross-sectional area (CSA) of the median nerve was measured at 2 points: (1) at the mid-forearm and; (2) just proximal to the carpal tunnel, where the nerve swelling was most prominent. The absolute value of the CSA at the point just proximal to the carpal tunnel and the ratio of the nerve swelling at this point to the mid-forearm point were used for analysis.

\section{Pressure Measurement}

Pressures were measured using the Spiegelberg intracranial pressure (ICP) monitoring device connected to a parenchymal type catheter (Spiegelberg GmbH \& Co., Hamburg, Germany).
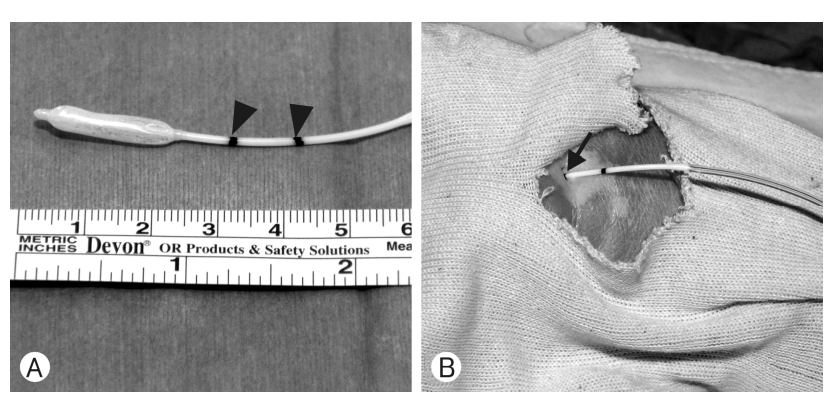

Fig. 1. Technique of carpal tunnel pressure measurement: (A) Spiegelberg intracranial pressure catheter is used for pressure measurement. The catheter shows 2 markers (arrowheads). (B) Location of the catheter tip is adjusted such that the first marker, which is located near the tip, is placed at the site of the skin incision. Note that the marker is visible at the site of the skin incision (arrow).
After dissection of the subcutaneous fat, the ligament was identified and incised in a transverse direction. Using surface landmarks as reference points, the Endocarp cannula was inserted so that the distal end of the cannula was located just distal to the distal end of the ligament. The placement of the cannula into the carpal tunnel was confirmed endoscopically. Subsequently, the ICP catheter was inserted through the cannula into the carpal canal. The depth of insertion was adjusted such that the first marker of the catheter was located at the skin incision site (Fig. 1).

\section{Assessment of Outcomes}

Treatment outcomes were assessed based on a 4-level scale at the time of last follow-up: (1) Excellent-patients free of symptoms; (2) Good-patients with mild residual symptoms without disturbances in using the hand; (3) Fair - patients with residual symptoms with some disturbance in using the hand; (4) Poor-patients with no improvement or the occurrence of postoperative complications.

\section{Statistical Analysis}

Data were analyzed using a commercially available statistical software package (SPSS version 20.0; IBM Corp., Armonk, NY, USA). Intergroup differences in variables were assessed using the $t$-test and the $X^{2}$ test. A p-value $<0.05$ was considered statistically significant.

\section{RESULTS}

\section{Demographic Characteristics}

We performed 304 operations in 247 patients $(57$ patients presented with bilateral CTS). We operated 220 women and 27 men. Among these patients, 39 (15.5\% of patients, 48 wrists) belonged to Group I. The median age of Group I was 74 years (range, 70-94 years). No significant difference was observed in the sex distribution between Groups I and II.

\section{NCS Grades}

All patients underwent EMG and NCS. Higher NCS grades was more common in Group I patients, and this difference was statistically significant ( $\mathrm{p}<0.0001, X^{2}$ test; Table 1 ). Thenar atrophy was observed in $25.0 \%$ of Group I patients (12/48) and in $12.1 \%$ of Group II patients (31/256). This difference was statistically significant $\left(\mathrm{p}=0.019, X^{2}\right.$ test).

\section{Assessment of Preoperative Clinical Symptoms}

Although the most common clinical symptom grade was 3 in both groups, a significant number of patients in Group 1 
showed higher grades of symptoms. The intergroup difference in distribution of symptom grades was statistically significant $\left(\mathrm{p}=0.018, X^{2}\right.$ test; Table 2$)$.

\section{USG and Assessment of CTP}

All patients underwent preoperative USG. The mean CSA of the median nerve at the carpal tunnel was $0.17 \pm 0.06 \mathrm{~cm}^{2}$ (mean \pm standard deviation [SD]) in Group I and $0.15 \pm 0.16 \mathrm{~cm}^{2}$ in Group II. Median nerve swelling was more severe in Group I ( $\mathrm{p}=0.04 ; t$-test). There was no statistically significant intergroup difference in the CSA of the median nerve at the forearm (Fig. 2). CTP was measured in 229 patients (283 wrists). CTP before division of the transverse carpal ligament was $39.3 \pm 20.4 \mathrm{mmHg}$ (mean $\pm \mathrm{SD}$ ) in Group I and $39.3 \pm 16.1 \mathrm{mmHg}$ in Group II. This pressure was reduced after division of the transverse carpal ligament $4.2 \pm 4.1 \mathrm{mmHg}$ in Group I and $4.2 \pm 3.7 \mathrm{mmHg}$ in Group II. No statistically significant intergroup difference in pressures was observed between Groups I and II before and

Table 1. Nerve conduction study grades

\begin{tabular}{lccccc}
\hline \hline & Negative & Mild & Moderate & Severe & Extreme \\
\hline Group I & 3 & 21 & 14 & 17 & 10 \\
Group II & 11 & 4 & 140 & 70 & 14 \\
\hline${ }^{*}$ Classification based on the study reported by Padua et al. $^{17)}$. \\
p $<0.0001$ & $\left(X^{2}\right.$ test).
\end{tabular}

Table 2. Clinical symptoms*

\begin{tabular}{llcccc}
\hline \hline & 1 & 2 & 3 & 4 & 5 \\
\hline Group I & 0 & 15 & 21 & 11 & 1 \\
Group II & 1 & 64 & 160 & 31 & 0 \\
\hline
\end{tabular}

${ }^{*}$ Historical-objective scale ${ }^{6)}$. $\mathrm{p}=0.018\left(X^{2}\right.$ test $)$.

Table 3. Treatment outcomes

\begin{tabular}{lcccc}
\hline \hline Outcome $^{*}$ & Excellent & Good & Fair & Poor \\
\hline Group I & 21 & 14 & 3 & 2 \\
Group II & 161 & 39 & 7 & 3 \\
\hline
\end{tabular}

"Please refer to materials and methods.

Table 4. The impact of age on outcome, nerve conduction study grade, clinical symptoms

\begin{tabular}{lccc}
\hline \hline Variable & Adjusted OR & $95 \% \mathrm{Cl}$ & p-value \\
\hline Age group & 2.93 & $1.48-5.81$ & 0.002 \\
NCS grade & 1.03 & $0.78-1.35$ & 0.828 \\
Hi-Ob scale & 1.26 & $0.79-1.99$ & 0.332 \\
\hline
\end{tabular}

NCS: nerve conduction study; OR: odds ratio; $\mathrm{Cl}$ : confidence interval, $\mathrm{Hi}$-Ob: historical-objective.

${ }^{*}$ Cumulative logistic regression. after division of the transverse carpal ligament (Fig. 3).

\section{Treatment Outcomes}

Open conversion was done in three cases (F/47, F/43, M/53) during endoscopic surgery due to persistent bulging bursa and masking of transverse carpal ligament. There was no case of postoperative complication.

Follow-up data (over $>4$ weeks postoperatively) were available in 205 patients (250 wrists).

Of the 40 wrists evaluated in Group I, 35 showed excellent and good results (87.5\%), whereas of the 210 wrists in Group II, 200 showed good results (95.2\%). This intergroup difference in treatment outcomes was statistically significant $\left(\mathrm{p}=0.005, X^{2}\right.$ test; Table 3). When we adjusted the difference in NCS grade

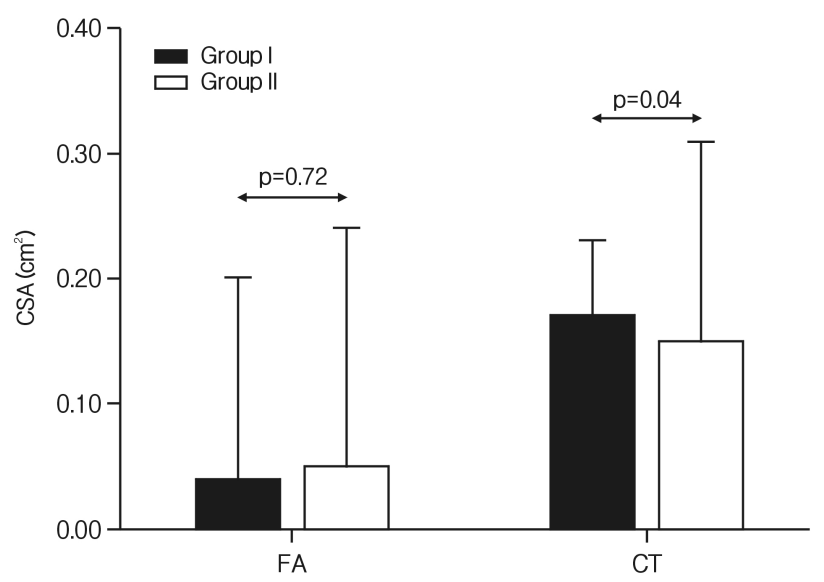

Fig. 2. Difference in cross sectional area (CSA) of median nerve measured with ultrasonography between Group I and II. No significant intergroup difference is observed in the CSA of the median nerve in the forearm (FA). However, the CSA of the median nerve is significantly larger in the carpal tunnel (CT) in Group I.

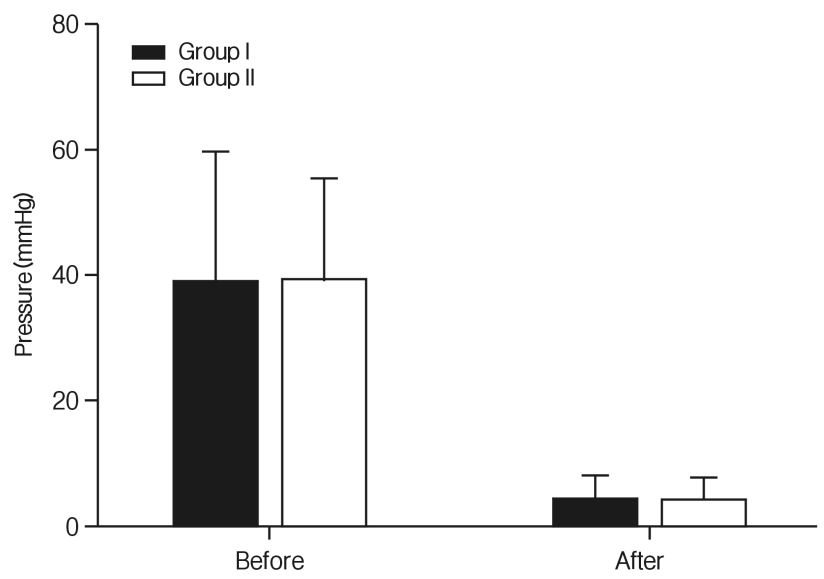

Fig. 3. Carpal tunnel pressure measurement: no significant intergroup difference is observed before and after division of the transverse carpal ligament. 
and clinical symptoms (Hi-Ob scale) between Group I and II, age showed significant impact on worse outcome (cumulative logistic regression; Table 4).

\section{DISCUSSION}

CTS is the most common entrapment neuropathy ${ }^{10)}$. Reportedly, the age-specific incidence for women gradually increases with age, peaks at the sixth decade and subsequently declines ${ }^{14)}$. The prevalence of CTS ranges between $1.3 \%$ and $2.0 \%$ in the general population among individuals aged $\geq 25$ years, and its incidence ranges between 0.3 and 3.3 per 1,000 person-year ${ }^{3}$. Reportedly, the incidence rate in middle-aged patients (40-49 years) and in patients aged $>70$ years is 2.8 and 1.4 per 1,000 person-year, respectively ${ }^{19)}$.

Differences in the clinical features and findings on EMG/NCS and USG between elderly and younger patients remain debatable. Reportedly, thenar atrophy is more common in the elderly group; however, the severity of clinical symptoms is debatable $^{4,23)}$. A few authors report no significant differences in clinical symptom scales, duration of symptoms, and the presence of autonomic features except thenar atrophy. Others report diurnal paresthesia is significantly confined to the elderly group. Our study showed that severe clinical symptoms (using the $\mathrm{Hi}-\mathrm{Ob}$ scale) and thenar atrophy were more common in Group I patients. A few authors suggest that the severity of symptoms might be related to the age-related decrease in peripheral nerve function, which is supported by the delay in the peripheral nerve conduction velocity in these patients ${ }^{9)}$.

In a series involving patients aged $>70$ years, $80 \%$ of the patients demonstrated electrophysiological study results indicating severe dysfunction of the median nerve ${ }^{25}$. Other studies have also reported similar results in elderly patients with CTS $^{9,20)}$. It is suggested that results of NCS indicating severe disease could be related to delayed treatment owing to age-related reduced pain sensitivity because of reduced nerve membrane excitability $^{21)}$. Reportedly, the CSA of the median nerve was correlated with distal motor and sensory latencies of the median nerve and severity of CTS except in patients aged $>80$ years in whom CSA was not $m$ regardless of severe CTS ${ }^{13)}$. Our study demonstrated that Group I patients showed higher NCS grades and USG proven more severe median nerve swelling.

Considering that the basic pathology of entrapment neuropathy is nerve compression, pressure on the nerve serves as the basic pathophysiological factor. It is known that the CTP was increased in CTS patients compared to normal controls, and the pressure dropped after the division of transverse carpal ligament ${ }^{1,16)}$. The reported correlation between CTP and severity in NCS suggested increased CTP underlie that the functional alteration assessed by nerve conduction ${ }^{8,12)}$. Our data showed no significant intergroup difference in CTP before and after division of the transverse carpal ligament. To our knowledge, this is the first series to compare the effect of pressure on the median nerve based on age. This suggests no significant intergroup difference in the basic pathology. The extent of postoperative pressure relief was the same in both groups, suggesting that the basic pathology had been reversed to the same extent in both groups. It might be argued that pressure measurements could differ depending upon the site of measurement within the carpal tunnel. As previously reported, the highest point is the point at which the catheter is inserted such that the first marker of the catheter is located at the site of the skin incision ${ }^{1)}$.

Treatment outcomes in elderly patients with CTS remain controversial. Although a few authors have reported satisfactory postoperative outcomes in elderly patients ${ }^{24)}$, others have reported poor postoperative outcomes in this population ${ }^{2,5,15,18)}$. Reportedly, functional status and symptom severity tend to show adequate postoperative improvement, although complete elimination of symptoms and restoration of function is unlikely ${ }^{28)}$. This might be related to the accelerated decline in perceived peripheral nerve function and age-related muscle changes. The poor outcomes could be related to delayed peripheral nerve regeneration and changes in the response of Schwann cells and macrophages to injury in elderly patients ${ }^{26)}$. It has also been shown that repair of segmental demyelination was nearly 2-fold slower in the aged group (5\% significance level), even when comparing groups with the same preoperative distal motor latency ${ }^{27)}$. Our data also showed poor outcomes in elderly patients. Because of the high frequency of higher NCS grade and more severe clinical symptoms, we adjusted its impact on outcome. The age showed significant impact on outcome after adjusting NCS grade and Hi-Ob scale, which means age is an independent prognostic factor (Table 4). Although the treatment outcome was poorer in Group I (87.5\%) than in Group II (95.2\%), it is comparable to the reported success rates for CTS operations $(72-94 \%)^{11)}$.

All operations in our case series were performed by a single surgeon using a single surgical technique. Thus, our data are homogeneous compared to previous studies in which several surgeons performed operations using various surgical methods. Limitations of our study are: (1) We used clinical scales for symptom and outcome assessments; however, our study did not use objective questionnaires such as the Boston Carpal Tunnel or the Michigan Hand Outcomes questionnaires owing to the retrospective study design. (2) Our follow-up period was relatively short, which would therefore not accurately reflect the true surgical outcome in elderly patients considering their slow regeneration potential as described above. (3) A few patients (particularly those with good results) were lost to follow-up because they did not return to our outpatient clinic. It might be speculated that patients with favorable outcomes are more likely to be lost to follow-up because they do not feel the need to re-visit the hospital after discharge.

\section{CONCLUSION}

Based on clinical signs, USG, and electrophysiological studies, 
we observed that the severity of CTS was higher in elderly than that in younger patients. Our study suggests no difference in basic preoperative pathology and reversal of that pathology postoperatively in terms of CTP. Despite the poorer outcomes in elderly patients, surgical treatment of CTS could be a reasonable treatment option considering that most patients in both groups were satisfied with the results. We conclude that the clinical, radiological, and electrodiagnostic features indicating a more severe disease presentation in Elderly patients are attributable to the reaction to compression of the median nerve in this age group.

\section{ACKNOWLEDGEMENT}

We thank Professor So-Hee Oh, Ph.D., Department of Biostati stics, Seoul National University Boramae Hospital for her technical support in statistical analysis.

\section{CONFLICT OF INTEREST}

No potential conflict of interest relevant to this article was reported.

\section{REFERENCES}

1. Ahn SY, Hong YH, Koh YH, Chung YS, Lee SH, Yang HJ: Pressure measurement in carpal tunnel syndrome: correlation with electrodiagnostic and ultrasonographic findings. J Korean Neurosurg Soc 46:199-204, 2009

2. Alrawashdeh O: Prevalence of asymptomatic neurophysiological carpal tunnel syndrome in 130 healthy individuals. Neurol Int 8:6553, 2016

3. Atroshi I, Gummesson C, Johnsson R, Ornstein E, Ranstam J, Rosén I: Prevalence of carpal tunnel syndrome in a general population. JAMA 282:153-158, 1999

4. Blumenthal S, Herskovitz S, Verghese J: Carpal tunnel syndrome in older adults. Muscle Nerve 34:78-83, 2006

5. Fakhouri F, Alsukhni RA, Altunbi B, Hawoot Z, Dabbagh R: Factors correlated with unfavorable outcome after carpal tunnel release surgery. Asian J Neurosurg 12:670-673, 2017

6. Giannini F, Cioni R, Mondelli M, Padua R, Gregori B, D'Amico $\mathrm{P}$, et al.: A new clinical scale of carpal tunnel syndrome: validation of the measurement and clinical-neurophysiological assessment. Clin Neurophysiol 113:71-77, 2002

7. Greenslade JR, Mehta RL, Belward P, Warwick DJ: Dash and Boston questionnaire assessment of carpal tunnel syndrome outcome: what is the responsiveness of an outcome questionnaire? J Hand Surg Br 29:159-164, 2004

8. Ikeda K, Osamura N, Tomita K: Segmental carpal canal pressure in patients with carpal tunnel syndrome. J Hand Surg Am 31: 925-929, 2006

9. Kanatani T, Nagura I, Kurosaka M, Kokubu T, Sumi M: Electrophysiological assessment of carpal tunnel syndrome in elderly patients: one-year follow-up study. J Hand Surg Am 39:21882191, 2014

10. Latinovic R, Gulliford MC, Hughes RA: Incidence of common compressive neuropathies in primary care. J Neurol Neurosurg Psychiatry 77:263-265, 2006

11. Louie D, Earp B, Blazar P: Long-term outcomes of carpal tunnel release: a critical review of the literature. Hand (N Y) 7:242-246, 2012

12. Luchetti R, Schoenhuber R, Nathan P: Correlation of segmental carpal tunnel pressures with changes in hand and wrist positions in patients with carpal tunnel syndrome and controls. J Hand Surg Br 23:598-602, 1998

13. Miwa T, Miwa H: Ultrasonography of carpal tunnel syndrome: clinical significance and limitations in elderly patients. Intern Med 50:2157-2161, 2011

14. Mondelli M, Giannini F, Giacchi M: Carpal tunnel syndrome incidence in a general population. Neurology 58:289-294, 2002

15. Mondelli M, Padua L, Reale F: Carpal tunnel syndrome in elderly patients: results of surgical decompression. J Peripher Nerv Syst 9:168-176, 2004

16. Okutsu I, Ninomiya S, Yoshida A, Hamanaka I, Kitajima I: Measurement of carpal canal and median nerve pressure in patients with carpal tunnel syndrome. Tech Hand Up Extrem Surg 8:124128, 2004

17. Padua L, Padua R, Nazzaro M, Tonali P: Incidence of bilateral symptoms in carpal tunnel syndrome. J Hand Surg Br 23:603606, 1998

18. Porter P, Venkateswaran B, Stephenson H, Wray CC: The influence of age on outcome after operation for the carpal tunnel syndrome. A prospective study. J Bone Joint Surg Br 84:688691, 2002

19. Pourmemari MH, Heliovaara M, Viikari-Juntura E, Shiri R: Carpal tunnel release: lifetime prevalence, annual incidence, and risk factors. Muscle Nerve [epub ahead of print, 2018. doi: 10.1002/mus.26145]

20. Povlsen B: High incidence of absent nerve conduction in older patients with bilateral carpal tunnel syndrome. Ann R Coll Surg Engl 92:403-405, 2010

21. Povlsen B, Aggelakis K, Koutroumanidis M: Effect of age on subjective complaints and objective severity of carpal tunnel syndrome: prospective study. JRSM Short Rep 1:62, 2010

22. Roh YH, Chung MS, Baek GH, Lee YH, Rhee SH, Gong HS: Incidence of clinically diagnosed and surgically treated carpal tunnel syndrome in Korea. J Hand Surg Am 35:1410-1417, 2010

23. Seror P: Carpal tunnel syndrome in the elderly. "Beware of severe cases”. Ann Chir Main Memb Super 10:217-225, 1991

24. Tomaino MM, Weiser RW: Carpal tunnel release for advanced disease in patients 70 years and older: does outcome from the patient's perspective justify surgery? J Hand Surg Br 26:481-483, 2001

25. Townshend DN, Taylor PK, Gwynne-Jones DP: The outcome of carpal tunnel decompression in elderly patients. J Hand Surg Am 30:500-505, 2005

26. Vaughan DW: Effects of advancing age on peripheral nerve regeneration. J Comp Neurol 323:219-237, 1992

27. Vyšata $\mathrm{O}$, Procházka A, Kunc $\mathrm{P}$, Kanta M, Ehler E, Yadollahi $\mathrm{M}$, et al.: Age delays the recovery of distal motor latency after carpal tunnel syndrome surgery. Acta Neurochir (Wien) 156: 1335-1339, 2014

28. Weber RA, DeSalvo DJ, Rude MJ: Five-year follow-up of carpal tunnel release in patients over age 65. J Hand Surg Am 35:207211, 2010 